\title{
OBESIDADE ASSOCIADA À DEFICIÊNCIA DE VITAMINA D EM PACIENTES COM INSUFICIÊNCIA CARDÍACA
}

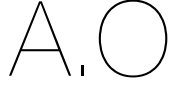

ARTIGO ORIGINAL

1 Instituto de Nutrição do

Cérebro e Coração, Estrada Francisco da Cruz Nunes,

n. ${ }^{\circ} 6723$ - sala 408

Niterói, Rio de Janeiro, Brasil

2 Universidade Federal

Fluminense,

R. Marquês de Paraná,

303 - Centro, Niterói, Rio

de Janeiro,

24070-035 Brasi

3 Faculdade de Medicina da Universidade Iguaçu, Av. Abílio Augusto Távora, n. 2134 - Dom Rodrigo, Nova lguaçu, Rio de Janeiro,

26260-045 Brasi

${ }^{4}$ Universidade Veiga de Almeida,

R. Ibituruna, n. ${ }^{\circ} 108$

Maracanã, Rio de Janeiro,

20271-020 Brasi

${ }^{5}$ Departamento de

Psiquiatria da Faculdade de

Medicina da Universidade

Federal Fluminense,

R. Marquês de Paraná,

○ 303 - Centro, Niterói,

Rio de Janeiro,

24070-035 Bras

${ }^{6}$ Departamento

de Cardiologia da

Faculdade de Medicina

da Universidade Federa

Fluminense,

R. Marquês de Paraná,

n. 303 - Centro, Niterói, Rio

de Janeiro,

24070-035 Brasil

7 Universidade Iguaçu, Av. Abílio Augusto Távora, n. 2 2134, Dom Rodrigo, Nova Iguaçu, Rio de

aneiro,

26260-045 Brasil

Endereço para correspondência: Thais Bessa-Guerra Estrada Francisco da Cruz Nunes, n. ${ }^{\circ} 6723$ - sala 408 , Niterói, Rio de Janeiro, Brasi crezende34@gmail.com

Histórico do artigo:

\author{
OBESITY ASSOCIATED WITH VITAMIN D DEFICIENCY IN \\ PATIENTS WITH HEART FAILURE
}

Thais Bessa-Guerra' ${ }^{1-3}$; Monyque Silva4; Dalmo de Lima5; Ana Cavalcanti²; Sandra Miranda²; Elisabeth Maróstica²; Mauro Mendlowicz; Evandro Mesquita ${ }^{a}$; Adalgiza Moreno

\section{RESUMO}

INTRODUÇÃo: A insuficiência cardíaca está associada a um grande número de fatores de risco, dentre eles a obesidade. Os fatores de risco modificáveis podem ser gerenciados para diminuir significativamente o risco para desenvolver a doença, como a investigação de biomarcadores metabólicos, como a vitamina D. No entanto, há poucas evidências nas diretrizes sobre a associação da vitamina D como fator de risco na população com insuficiência cardíaca.

OBJETIVOS: Avaliar níveis de vitamina $\mathrm{D}$ como fator de risco associado à obesidade em pacientes com insuficiência cardíaca. METODOLOGIA: Estudo observacional, prospetivo, tipo caso-controlo, realizado em uma clínica multiprofissional especializada em insuficiência cardíaca. O diagnóstico do estado nutricional foi realizado através do questionário validado miniavaliação nutricional (MAN) e antropometria. A coleta de sangue foi realizada seguindo as recomendações de biossegurança. A distribuição dos dados fol avaliada pelos testes de Kolmogorov-Smirnov e Shapiro-Wilk. Foi considerado significativo valor de $p<0,05$. A análise estatística foi feita através do Software Statistical Package for the Social Sciences, versão 20.0.

RESULTADOS: Avaliou-se 76 pacientes, 51,3\% com classe funcional II (New York Heart Association). Em relação ao estado nutricional $58 \%$ apresentavam score de risco (MAN) e 75,1\% com sobrepeso e obesidade; os níveis séricos de vitamina D são significativamente menores no grupo com obesidade ( $80 \%$; $p=0,003)$; as medidas de circunferência de cintura são significativamente maiores no grupo com obesidade ( $p=0,020)$; os scores do risco nutricional (MAN) são significativamente maiores no grupo com obesidade ( $<<0,001)$. A insuficiência de vitamina D aumentou a chance para desenvolver obesidade (OR:4,5; IC95\%:[1,1-19,2]).

CONCLUSÕES: A investigação permitiu detetar que a insuficiência de vitamina $D$ aumenta a chance para desenvolver obesidade em pacientes com insuficiência cardíaca.

\section{PALAVRAS-CHAVE}

Avaliação nutricional, Estado nutricional, Insuficiência cardíaca, Obesidade, Sobrepeso, Vitamina D

\section{ABSTRACT}

INTRODUCTION: Heart failure is associated with a large number of risk factors, including overweight, obesity and stress. Modifiable risk factors can be managed to significantly decrease the risk of developing the disease, such as investigating metabolic biomarkers, such as vitamin D. However, there is little evidence in the guidelines on the association of vitamin D as a risk factor in the population with heart failure.

OBJECTIVES: To evaluate vitamin D levels as a risk factor associated with obesity in patients with heart failure. METHODOLOGY: Observational, cross-sectional and prospective study carried out in a multiprofessional clinic specialized in heart failure. The diagnosis of nutritional status was performed using the validated mini-nutritional assessment questionnaire (MNA), anthropometry. Blood collection was carried out following biosafety recommendations. Data distribution was assessed using the Kolmogorov-Smirnov and Shapiro-Wilk tests. A p value $<0.05$ was considered significant. Statistical analysis was performed using the Software Statistical Package for the Social Sciences, version 20.0.

RESULTS: 76 patients were evaluated, 51.3\% with functional class II (New York Heart Association). Regarding nutritional status, 58\% had a risk score (MAN) and $75.1 \%$ were overweight and obese; serum vitamin D levels are significantly lower in the obese group (80\%; $p=0.003)$; waist circumference measurements are significantly higher in the obese group ( $p=0.020)$; nutritional risk scores (MAN) are significantly higher in the group with obesity $(p<0,001)$. Vitamin $D$ insufficiency increased the chance of developing obesity (OR: 4.5; 95\% Cl: [1,1-19,2])

CONCLUSIONS: The investigation made it possible to identify a phenotype with a higher risk and was useful for detecting vitamin D insufficiency as a risk factor associated with obesity in outpatients with heart failure.

\section{KEYWORDS}

Nutritional assessment, Nutritional status, Cardiac insufficiency, Obesity, Overweight, D vitamin 


\section{INTRODUÇÃO}

A insuficiência cardíaca (IC) é uma síndrome multissistêmica de caráter progressivo, com complexa interação entre fatores genéticos, neurohormonais, inflamatórios e metabólicos, que frequentemente tem sido associada com as multi-morbidades, dentre elas a obesidade (1, 2). A IC está associada a um grande número de fatores de risco, incluindo a falta de atividade física, uma dieta insalubre, uso intenso de álcool, stresse, excesso de peso ou obesidade e insuficiência de vitamina $D$ $(1,2,3,4)$. Recentemente, um estudo mostrou que a deficiência de vitamina $D$ foi identificada como um fator de risco modificável para IC, aumentando o risco para 4,18 vezes mais provável em indivíduos com obesidade do que aqueles com peso normal (4).

Novos estudos vêm se concentrando na descoberta de elementos que possam contribuir para a patogénese da IC na presença da obesidade. Dentre eles, vem sendo estudado o papel da vitamina $D$, mais precisamente a sua deficiência e o efeito da sua suplementação tem chamado sua atenção para patogenia da IC (5).

Portanto, investigar em pacientes ambulatoriais com IC representa uma janela de oportunidade para conhecermos os fatores de riscos associados à obesidade e suas vias de sinalização para síntese da vitamina $\mathrm{D}$ e dos biomarcadores inflamatórios e metabólicos. Nesse contexto, este estudo tem como objetivo avaliar os níveis séricos de vitamina $\mathrm{D}$ associados com a obesidade em pacientes ambulatoriais com IC.

\section{METODOLOGIA}

Estudo observacional, prospetivo, caso-controlo, seguindo os critérios estabelecidos pela Strengthening the Reporting of Observacional Studies in Epidemiology (STROBE) (6), em pacientes ambulatoriais com IC atendidos na clínica multiprofissional especializada.

\section{Aspetos Éticos}

Este estudo foi aprovado pelo Comité de Ética sob o parecer n. ${ }^{\circ}$ 630.078, sob o n CAAE: 25093513.0.0000.5243 da Universidade Federal Fluminense. Todos os participantes assinaram o Termo de Consentimento Livre e Esclarecido.

\section{Seleção da Amostra}

A amostra foi selecionada por conveniência, seguindo os critérios de diagnóstico para IC através do método de Simpson. Para os critérios de inclusão foram considerados adultos e idosos classe funcional I, II ou III segundo os critérios de classificação da New York Heart Association (NYHA), mínimo de participação de uma consulta nos últimos 6 meses; capacidade de comunicação preservada. Os critérios de exclusão foram considerados NYHA IV, défice cognitivo registrado em prontuário, uso de antidepressivos, pelo viés da reclusão, o que certamente impactaria nas rotinas diárias e exposição aos raios solares.

\section{Protocolo do Estudo}

Para avaliação do estado nutricional utilizou-se o questionário Mini Avaliação Nutricional (MAN) (7), classificado: normal (ou sem risco de desnutrição), quando a pontuação for acima de 23,5 pontos, em risco nutricional, quando a pontuação for entre 17 a 23,5 ou desnutrido, quando a pontuação for menor que 17 pontos $(7,8)$.

As medidas antropométricas foram realizadas seguindo a padronização da World Health Organization (WHO) (9), preferencialmente pela manhã, em duplicatas e por único examinador. O IMC foi calculado pela razão peso $(\mathrm{kg}) /$ altura $\left(\mathrm{m}^{2}\right)$ e classificados segundo os valores de referência da WHO (9). As medidas de Circunferência de Cintura (CC) foram mensuradas, considerando o ponto de corte para homens $>=102 \mathrm{~cm}$ e mulheres $>=88 \mathrm{~cm}(9)$.
Para coleta das amostras de sangue foi realizado jejum de 12 horas. Para a realização da análise da vitamina D o material foi preparado no equipamento da marca SIGMA ${ }^{\circledR}$ a 3.000 rotações por minuto, durante 15 minutos. Foi utilizado o Kit da Marca Siemens Healthcare Diagnostics Inc ${ }^{\circledR}$, Ref: RF642, realizado pelo método de Quimioluminescência. Os valores de referências considerados foram: Suficiência de 30 a 100 ng/mL; Insuficiência de 10 a 29 ng/mL; Insuficiência inferior a $10 \mathrm{ng} / \mathrm{mL}$; Toxicidade- Superior a $100 \mathrm{ng} / \mathrm{mL}$ (10).

\section{Análise Estatística}

A análise descritiva caracterizou a população com frequências e proporções. Para as variáveis quantitativas, foram utilizadas as estatísticas de valores mínimo, máximo, média, desvio padrão, mediana e coeficiente de variação (CV). Para investigar a significância da associação entre duas variáveis qualitativas, foi utilizado o teste do qui-quadrado e, quando este se mostrou inconclusivo, foi realizado o teste exato de Fisher para tabelas 2×2 (9). A hipótese de normalidade foi verificada pelos testes de Kolmogorov Smirnov (KS) e de ShapiroWilk (SW). Para comparar dois grupos independentes com variáve com distribuição normal, foi utilizado o teste $t$ de Student não pareado; caso contrário, a comparação entre dois grupos foi feita pelo teste não paramétrico de Mann-Whitney (9). A medida usada para expressar o risco foi a Razão de Chances ou Odds Ratio (OR), com significância da OR foi avaliada pelo intervalo da OR, que não pode conter o valor 1. A análise estatística foi realizada com o programa Statistical Package for the Social Sciences (SPSS), versão 20.0, e todas as análises foram realizadas considerando um nível de significância máximo de 5,0\%.

\section{RESULTADOS}

Foram avaliados por amostra de conveniência para elegibilidade 85 pacientes. Excluídos 09, por uso de antidepressivos $(n=4)$; défice cognitivo ( $n=2)$; nível de escolaridade analfabeto $(n=3)$, sendo eleitos prospetivamente $\mathrm{n}=76$.

Participaram deste estudo 76 pacientes, sendo 40 (52,6\%) do sexo feminino e $36(47,4 \%)$ do sexo masculino. Em relação às características socioeconómicas a renda mensal dos pacientes não seguiu uma distribuição normal ( $p<0,001)$, e variou entre $R \$ 500,00$ e 3.000,00, com uma média de $R \$ 1.118,74$. Os pacientes eram de cor autodeclarada branca (67,1\%), nível de escolaridade de ensino fundamental I (56,6\%). Em relação às características clínicas os pacientes apresentavam classe funcional NYHA II (51,3\%), hipertensão (100\%), dislipidemia $(81,6 \%)$, diabetes $(56,4 \%)$, sobrepeso e obesidade $(75,1 \%)$. Não havia diferença significativa entre a idade de homens e mulheres $(p=0,056)$, nem entre a renda de homens e mulheres $(p=0,644)$. Os grupos feminino e masculino não diferiram em relação à distribuição de cor autodeclarada $(p=0,641)$, escolaridade $(p=0,352)$ e incidência de diabetes $(p=0,223)$, dislipidemia $(p=0,827)$, insuficiência renal crónica (IRC; $p=0,426)$, doença pulmonar obstrutiva crónica (DPOC; $p=0,601$ ) e acidente vascular encefálico (AVE, $p=1,000$ ) (Tabela 1).

Observou-se alta variabilidade nas medidas dos biomarcadores cardiometabólicos entre os pacientes. As diferenças das distribuições das variáveis: vitamina $\mathrm{D}, \mathrm{CC}$ e score do risco nutricional (MAN) nos grupos sem obesidade e com obesidade foram significativas. Observou-se que os níveis séricos de Vitamina D são significativamente menores no grupo $<30$ ( $p=0,003)$; as medidas de CC são significativamente maiores no grupo com obesidade $(p=0,020)$ (Tabela 2$)$.

Os resultados da associação da obesidade com a insuficiência de Vitamina D mostram que a proporção de pacientes sem obesidade com insuficiência de vitamina D é de $47,1 \%$. No grupo com obesidade essa 
Tabela 1

Características sócio-demográficas e clínicas em pacientes ambulatoriais com insuficiência cardíaca

\begin{tabular}{|c|c|c|}
\hline VARIÁVEL & $\mathrm{n}$ & $\%$ \\
\hline \multicolumn{3}{|l|}{ Sexo } \\
\hline Feminino & 40 & $52,6 \%$ \\
\hline Masculino & 36 & $47,4 \%$ \\
\hline \multicolumn{3}{|l|}{ Cor autodeclarada } \\
\hline Branca & 51 & $67,1 \%$ \\
\hline Preta & 22 & $28,9 \%$ \\
\hline Indeterminada & 03 & $3,9 \%$ \\
\hline Idade (média \pm DP) & & $63,03 \pm 13,5$ anos \\
\hline \multicolumn{3}{|l|}{ Nível de Escolaridade } \\
\hline Alfabetizado & 2 & $2,6 \%$ \\
\hline Ensino fundamental I & 43 & $56,6 \%$ \\
\hline Ensino Fundamental II & 28 & $36,8 \%$ \\
\hline Ensino Médio & 3 & $3,9 \%$ \\
\hline Renda familiar em reais (média \pm DP) & & $\mathrm{R} \$ 1.118,74 \pm 576,32$ \\
\hline \multicolumn{3}{|l|}{ Classe Funcional (NYHA) } \\
\hline NYHA I & 23 & $30,3 \%$ \\
\hline NYHA II & 39 & $51,3 \%$ \\
\hline NYHA III & 14 & $18,4 \%$ \\
\hline \multicolumn{3}{|l|}{ Comorbidades } \\
\hline Hipertensão Arterial Sistémica & 76 & $100,0 \%$ \\
\hline Dislipidemia & 62 & $81,6 \%$ \\
\hline Diabetes Mellitus & 43 & $56,6 \%$ \\
\hline \multicolumn{3}{|l|}{ IMC (kg/m²) } \\
\hline Baixo peso & 1 & $1,3 \%$ \\
\hline Peso normal & 18 & $23,7 \%$ \\
\hline Sobrepeso & 11 & $14,5 \%$ \\
\hline Pré-obesidade & 12 & $15,8 \%$ \\
\hline Obesidade I & 23 & $30,3 \%$ \\
\hline Obesidade II & 7 & $9,2 \%$ \\
\hline Obesidade III & 4 & $5,3 \%$ \\
\hline Insuficiência Renal Crónica & 18 & $23,7 \%$ \\
\hline Anemia & 15 & $19,7 \%$ \\
\hline Acidente Vascular Encefálico & 4 & $5,3 \%$ \\
\hline Doença Pulmonar Obstrutiva Crónica & 3 & $3,9 \%$ \\
\hline Cancro & 2 & $2,6 \%$ \\
\hline Hepatite & 1 & $1,3 \%$ \\
\hline Hipotireoidismo & 1 & $1,3 \%$ \\
\hline Hipertireoidismo & 1 & $1,3 \%$ \\
\hline \multicolumn{3}{|l|}{ Classificação da Insuficiência Cardíaca } \\
\hline ICFEP & 42 & $56,0 \%$ \\
\hline ICFER & 33 & $44,0 \%$ \\
\hline
\end{tabular}

DP: Desvio-padrão

ICFEP: Insuficiência cardiaca com fração de ejeção preservada

ICFER: Insuficiência cardiaca com fração de ejeção reduzida

IMC: Índice de Massa Corporal

NYHA: New York Heart Association

proporção é significativamente maior, igual a 80,0\%. A insuficiência de vitamina $\mathrm{D}$ constitui um fator de risco associado à obesidade, a razão de chances é igual a 4,5 com intervalo de confiança significativo (IC 95\% [1,1;19,2]). Estima-se que a chance de um obeso ter insuficiência de vitamina $D$ é 4,5 vezes a chance de um não obeso apresentar insuficiência de vitamina D (Tabela 3).

\section{DISCUSSÃO DOS RESULTADOS}

Este é o primeiro estudo que analisou o risco da insuficiência de vitamina $\mathrm{D}$ associado com a obesidade em pacientes ambulatoriais em uma clínica multiprofissional especializada em IC do Sistema Único de Saúde (SUS). A vitamina D não tem sido analisada sistematicamente na presença da obesidade em pacientes com IC $(5,12)$. A relação entre deficiência de vitamina D e obesidade aparentemente estão relacionadas entre si, embora ainda se desconheçam quais são os mecanismos de causa e efeito desta associação. Um dos mecanismos é pelo fato da vitamina $D$ ser lipossolúvel, o que faz com que ocorra uma maior captação de vitamina D pelo tecido adiposo $(5,10,11,12)$. Os principais achados nesses estudos mostram elevado percentual de sobrepeso e obesidade ( $\left(\mathrm{MC} \geq 25 \mathrm{Kg} / \mathrm{m}^{2}\right)$ em pacientes com IC. A Diretriz Brasileira de IC (2), evidenciou que cerca de $29 \%$ a $40 \%$ destes pacientes estão com sobrepeso, 30\% a 49\% estão obesos. No entanto, o conceito do paradoxo da obesidade na IC tem sido debatido por vários outros estudos, mas não associado com a insuficiência de vitamina $D(2,12,14)$. Estudos sugerem que os adipócitos possuem recetores de vitamina D, e que a vitamina D desempenha um papel na regulação da lipólise e 
Tabela 2

Associação de variáveis quantitativas com a Obesidade

\begin{tabular}{|c|c|c|c|c|c|c|c|}
\hline \multirow{2}{*}{ VARIÁVEL } & \multicolumn{3}{|c|}{ SEM OBESIDADE } & \multicolumn{3}{|c|}{ COM OBESIDADE } & \multirow{2}{*}{ p-VALOR } \\
\hline & MÉDIA & DP & MEDIANA & MÉDIA & DP & MEDIANA & \\
\hline Idade & 63,1 & 11,1 & 63,0 & 62,9 & 12,4 & 63,1 & 0,942 \\
\hline Renda & 1171,9 & 631,2 & 1000,0 & 1054,5 & 500,9 & 950,0 & 0,595 \\
\hline ICFER & 51,3 & 16,9 & 50,0 & 59,1 & 15,7 & 61,0 & 0,061 \\
\hline Vitamina D (ng/dL) & 35,5 & 12,3 & 30,2 & 25,4 & 6,2 & 26,4 & 0,003 \\
\hline Uréia (mg/dL) & 41,4 & 16,0 & 42,0 & 37,9 & 17,9 & 38,5 & 0,257 \\
\hline Creatinina (mg/dL) & 1,1 & 0,4 & 1,1 & 1,0 & 0,4 & 0,9 & 0,341 \\
\hline Sódio sérico (mg/dL) & 139,5 & 4,2 & 140,0 & 137,9 & 2,1 & 138,0 & 0,062 \\
\hline Potássio (mg/dL) & 4,5 & 0,5 & 4,5 & 4,6 & 0,8 & 4,4 & 0,731 \\
\hline Glicose (mg/dL) & 106,0 & 24,0 & 101,0 & 115,1 & 43,1 & 103,0 & 0,749 \\
\hline Hemoglobina glicosilada(mg/dL) & 6,4 & 1,3 & 6,1 & 6,3 & 1,3 & 5,7 & 0,831 \\
\hline Colesterol total(mg/dL) & 163,3 & 41,4 & 162,5 & 170,6 & 40,6 & 169,0 & 0,287 \\
\hline LDL (mg/dL) & 91,9 & 37,8 & 88,0 & 99,7 & 37,4 & 105,5 & 0,266 \\
\hline $\mathrm{HDL}(\mathrm{mg} / \mathrm{dL})$ & 43,4 & 17,7 & 41,0 & 44,1 & 13,9 & 41,0 & 0,838 \\
\hline VLDL (mg/dL) & 29,7 & 16,8 & 25,0 & 25,3 & 16,6 & 20,5 & 0,243 \\
\hline Triglicerídeos (mg/dL) & 139,5 & 77,6 & 125,0 & 123,5 & 67,5 & 101,0 & 0,401 \\
\hline Ácido úrico (mg/dL) & 6,6 & 1,7 & 6,4 & 6,1 & 1,8 & 5,9 & 0,366 \\
\hline Magnésio (mg/dL) & 2,1 & 0,3 & 2,0 & 2,1 & 0,3 & 2,0 & 0,892 \\
\hline Circunferência da Cintura & 94,2 & 11,9 & 94,0 & 102,1 & 14,1 & 100,5 & 0,020 \\
\hline Score: Risco Nutricional (MAN) & 20,9 & 3,1 & 20,5 & 23,3 & 2,7 & 23,5 & 0,001 \\
\hline
\end{tabular}

Teste T-student não pareado ou teste de Mann Whitney

(\%) percentual

DP: Desvio-padrão

FE: Fração de ejeção

ICFER: Insuficiência cardíaca com fração de ejeção reduzida

LDL: Low Density Lipoprotein

MAN: Mini avaliação nutricional

VLDL: Very low density lipoproteins

HDL: High Density Lipoprotein

Tabela 3

Características clínicas nos grupos sem obesidade e com obesidade em pacientes com insuficiência cardíaca

\begin{tabular}{|c|c|c|c|c|c|c|c|}
\hline \multirow{2}{*}{ FATOR } & \multicolumn{2}{|c|}{$\begin{array}{c}\text { INCIDÊNCIA DO FATOR } \\
I M C<30\end{array}$} & \multicolumn{2}{|c|}{$\begin{array}{c}\text { INCIDÊNCIA DO } \\
\text { IMC >= } 30\end{array}$} & \multirow{2}{*}{ p-VALOR } & \multirow{2}{*}{ OR } & \multirow{2}{*}{ IC95\% DA OR } \\
\hline & $\mathrm{n}$ & $(n=42)$ & n & $\mathrm{n}=34$ ) & & & \\
\hline Sexo masculino & 22 & $52,4 \%$ & 14 & $41,2 \%$ & 0,331 & & $0,3-1,6$ \\
\hline Diabetes Melittus & 25 & $59,5 \%$ & 18 & $52,9 \%$ & 0,565 & 0,8 & $0,3-1,9$ \\
\hline Dislipidemia & 32 & $76,2 \%$ & 30 & $88,2 \%$ & 0,178 & 2,3 & $0,7-8,3$ \\
\hline Anemia & 10 & $23,8 \%$ & 5 & $14,7 \%$ & 0,321 & 0,6 & $0,2-1,8$ \\
\hline IRC & 10 & $23,8 \%$ & 8 & $23,5 \%$ & 0,977 & 1,0 & $0,3-2,9$ \\
\hline ICFER & 16 & $48,5 \%$ & 8 & $27,6 \%$ & 0,092 & 0,4 & $0,14-1,17$ \\
\hline Risco Nutricional (MAN) & 22 & $52,4 \%$ & 21 & $61,8 \%$ & 0,412 & 1,5 & $0,6-3,7$ \\
\hline Insuficiência de Vitamina D (ng/dL) & 8 & $47,1 \%$ & 16 & $80,0 \%$ & 0,036 & 4,5 & $1,1-19,2$ \\
\hline Creatinina $(\mathrm{mg} / \mathrm{dL})$ & 7 & $22,6 \%$ & 8 & $29,6 \%$ & 0,541 & 1,4 & $0,4-4,7$ \\
\hline
\end{tabular}

ICFER: Insuficiência cardíaca com fração de ejeção reduzida

IRC: Insuficiência renal crónica

MAN: Mini avaliação nutricional

que a forma ativa dessa vitamina poderia regular a morte de adipócitos e diminuição de massa gorda $(12,14,15,16)$.

As células de gordura agem como um depósito de grande capacidade para o armazenamento e liberação de vitamina $D$, acumulando a vitamina $D$ proporcionalmente à sua concentração no soro, e liberando-a de forma muito mais lenta, devido à grande quantidade de gordura. Isto pode afetar a biodisponibilidade da vitamina $\mathrm{D}$ e prejudicar sua atividade biológica $(11,14,17)$. Outro fator que pode estar envolvido na associação da deficiência dessa vitamina com a obesidade é a menor conversão de vitamina D3 em 25OHD no fígado, como consequência da presença de esteatose hepática não alcoólica (EHNA) em pessoas obesas $(12,14,17,18)$.

Outro achado com prevalência relevante em pacientes com IC foram os elevados percentuais de comorbidades clínicas, como diabetes, dislipidemia e Insuficiência Renal Crónica (IRC). As atuais evidências relatam que as doenças sistémicas e hipovitaminose $\mathrm{D}$ contribuem para cronicidade da IC e tem sido associada com as doenças inflamatórias crónicas, incluindo diabetes e obesidade. A vitamina $\mathrm{D}$ parece atuar também no controlo da secreção de insulina e controle dos níveis pressóricos (17-20).

A deficiência de vitamina $D$ se associa à $I C$ e a suplementação de vitamina $D$ pode ser benéfica (20). No entanto, há relatos de que não há melhora ou efeito benéfico com a suplementação da vitamina D na IC e as discrepâncias entre os estudos podem ser devido às variações genéticas do gene VDR $(12,14,21,22,23)$. Já, no estudo VINDICATE mostrou melhoria e efeitos benéficos da vitamina $D$ na estrutura e função do ventrículo esquerdo em pacientes com IC crónica em tratamento de vitamina $D$ (24) e diminuição da atividade da renina com suplementação de vitamina $\mathrm{D}$ e a curto prazo em pacientes com IC crónica sugerem o papel terapêutico da vitamina D (24). 
Em relação ao estado nutricional dos pacientes com IC, Joaquin et al (2019) (27) relatou no estudo de revisão que não há consenso sobre o melhor método de triagem e avaliação nutricional com MAN em pacientes com IC foi capaz de detectar o risco nutricional, sendo considerado marcador prognóstico. Em outro estudo (28) que utilizou MAN para avaliar a desnutrição em pacientes hospitalizados com IC corrobora com a média de idade dos pacientes do nosso estudo quanto com a prevalência do risco nutricional.

No entanto, nosso estudo destaca-se em relação ao estado nutricional de pacientes ambulatoriais avaliados pelo MAN quando comparado com outros estudos a nível hospitalar, isso traz a luz sobre a questão subestimada dessa avaliação, devido à falta de métodos padronizados de diagnóstico nas diretrizes de IC. Inclusive, nossos achados mostram que o elevado percentual do risco nutricional associado com a obesidade, é uma necessidade de discussão, devido à escassez de evidências sobre o diagnóstico do risco nutricional em pacientes com IC (3, 24-26).

Ainda assim, este estudo apresenta limitações pelo delineamento observacional e transversal, que não nos permitiu estabelecer as variáveis preditoras na IC. Porém, através dos dados apresentados fica evidente a importância desta discussão.

\section{CONCLUSÕES}

Baseado nos resultados deste estudo sobre os níveis de vitamina $D$ associados com a obesidade em pacientes com IC, encontramos os seguintes achados: 1) a sobrepeso e obesidade apresentam prevalência relevante em pacientes ambulatoriais com IC; 2) score do risco nutricional são significativamente maiores no grupo com obesidade em pacientes ambulatoriais com IC; 3) os níveis séricos de vitamina $D$ são significativamente menores no grupo com obesidade em pacientes ambulatoriais com IC.

Esta pesquisa demonstra a necessidade de futuros trabalhos adicionais sobre a vitamina $\mathrm{D}$ associada com a obesidade em pacientes com IC, em função da relevante prevalência e dos prejuízos desta associação.

\section{REFERÊNCIAS BIBLIOGRÁFICAS}

1. Rohde $L$ et al. Comitê Coordenador da Diretriz de Insuficiência Cardíaca. Diretriz Brasileira de Insuficiência Cardíaca Crônica e Aguda. Arq Bras Cardiol. 2018; 111:436539. Disponível: http://publicacoes.cardiol.br/portal/abc/portugues/2018/v11103/ pdf/11103021.pdf.

2. Mesquita ET et al. Entendendo a Hospitalização em Pacientes com Insuficiência Cardíaca. International Journal of Cardiovascular Sciences. 2017; 30:81-90.

3. Bessa-Guerra TR. O paradigma da psiquiatria nutricional associado aos diferentes métodos de rastreamento da depressão em pacientes com insuficiência cardíaca. Universidade Federal Fluminense. Tese de Doutorado. 2018; 137. Disponível: https:// app.uff.br/riuff/handle/1/8865.

4. Porto C. Associação entre deficiência de vitamina de riscos cardiovascular e de insuficiência cardíaca em idosos. Dissertação: associação entre deficiência de vitamina D e riscos cardiovascular e de insuficiência cardíaca em idosos. Universidade Federal de Pernambuco. 2016. Disponível: https://repositorio.ufpe.br/handle/123456789/17956. 5. Montera VSP, Mesquita ET. O Papel da Vitamina D na Insuficiência Cardíaca. Rev Bras Cardiol. 2010; 23:124-130.

6. Ebrahim S, Clarke M. STROBE: new standards for reporting observational epidemiology, a chance to improve. Int J Epidemiol. 2007; 36: 946-8.

7. Guigoz Y, Vellas B, Garry PJ. Mini nutritional assessment: A practical assessment tool for grading the nutritional state of elderly patients. Facts and Research in Gerontology. 1994, 2: 15-59.

8. Acuña KCT. Avaliação do estado nutricional de adultos e idosos e situação nutricional da população brasileira. Arq Bras Endocrinol Metab. 2004; 48: 345-361.
9. World Health Organization. Obesity and overweight. 2020. Disponível em: https:// www.who.int/news-room/fact-sheets/detail/obesity-and-overweight.

10. Maeda $S$ et al. Recomendações da Sociedade Brasileira de Endocrinologia e Metabologia (SBEM) para o diagnóstico e tratamento da hipovitaminose D. Arq Bras Endocrinol Metab [Internet]. 2014; 58(5): 411-433. Disponível: https://www.scielo.br/ scielo.php?script=sci_arttext\&pid=S0004-27302014000500411.

11. Pagano M, kimberlee G. Princípios de Bioestatística. 2004. São Paulo: Pioneira Thonson Learning.

12. Holick MF. Vitamin D deficiency. N Eng J Med. 2007; 357(3):266-81. PMID: 17634462.

13. Rai V, Agrawal DK. Papel da vitamina D nas doenças cardiovasculares. Endocrinol Metab Clin North Am. 2017; 46: 1039-1059.

14. Zamora E et al. Weight Loss in Obese Patients With Heart Failure. J Am Heart Assoc. 2016; 24:5.

15. Schmidt $A$. Relação entre a deficiência de vitamina $D$ e obesidade: uma revisão atual. Revista Brasileira de Obesidade, Nutrição e Emagrecimento. 2015; 9: 207-212. ISSN 1981-9919.

16. Barchetta I, De Bernardinis M, Capoccia D, Baroni MG, Fontana M. Hypovitaminosis $\mathrm{D}$ is Independently Associated with Metabolic Syndrome in Obese Patients. PLoS ONE. 2013; 8.

17. Targher G, Bertolini L, Scala L. Associations between serum 25- hydroxyvitamin D3 concentrations and liver histology in patients with non-alcoholic fatty liver disease. Nutr Metab Cardiovasc Dis. 2007; 17:517-524.

18. Paschou $S$ et al. O Impacto da Obesidade na Associação entre Deficiência de Vitamina D e Doenças Cardiovasculares. Nutrientes. 2019; 11: 2458.

19. Monteiro E, Baptista N, Faria A, Loureiro H. Deficiência de vitamina D em crianças com doença celíaca. Acta Portuguesa de Nutrição. 2019: 56-59.

20. Heaney R, Dowell M, Hale C. Calcium absorption varies within the reference range for serum 25-hydroxyvitamin D. J Am Coll Nutr. 2003; 22: 142-146.

21. Jiang W, Gu H, Zhang Y, Xia Q, Qi J, Chen J. Suplementação de vitamina D no tratamento da insuficiência cardíaca crônica: uma meta-análise de ensaios clínicos randomizados. Clin Cardiol. 2016; 39: 56-6.

22. Rai V, Agrawal DK. Role of Vitamin D in Cardiovascular Diseases. Endocrinology and Metabolism Clinics of North America. 2017; 46 (4):1039-1059. DOI: 10.1016/j. ecl.2017.07.009.

23. Siqueira T, Araujo J, Mattar R, DAHER S. Assessment of Polymorphism of the VDR Gene and Serum Vitamin D Values in Gestational Diabetes Mellitus. Rev. Bras. Ginecol. Obstet. [online]. 2019; 41: 425-431. Disponível: http://dx.doi. org/10.1055/s-0039-1693678.

24. Witte KK et al. Effects of Vitamin D on Cardiac Function in Patients With Chronic HF: The VINDICATE Study. J Am Coll Cardiol. 2016; 67(22):2593-603. doi: 10.1016/j. jacc.2016.03.508.

25. Schroten NF et al. Short-term vitamin D3 supplementation lowers plasma renin activity in patients with stable chronic heart failure: an open-label, blinded end point, randomized prospective trial (VitD-CHF trial). Am Heart J. 2013; 166(2):357-64.

26. Ruiz, JM. Impacto de la valoración nutricional en los pacientes com insuficiência cardíaca. Nutr Hosp.2017; 34(6): 12656.

27. Raslan M. et al. Risco nutricional e complicações em obesos hospitalizados submetidos à cirurgia. ABCD, Arq. bras. cir. dig. [online]. 2007; 20:261-265. 2007. Disponivel: https://doi.org/10.1590/S0102-67202007000400010.

28. Joaquín C, Puig R, Gastelurrutia P, et al. Mini nutritional assessment is a better predictor of mortality than subjective global assessment in heart failure out-patients. [online]. Clin Nutr. 2019; 38(6): 2740-2746. Disponível: https://pubmed.ncbi.nlm.nih. gov/30616882/.

29. Kałuzna-Oleksy M et al. Relationship between Nutritional Status and Clinical and Biochemical Parameters in Hospitalized Patients with Heart Failure with Reduced Ejection Fraction, with 1-year Follow-Up. Nutrients. 2020; 12: 2330. Disponível: https:// www.ncbi.nlm.nih.gov/pmc/articles/PMC7468814/. 